\title{
Viscous-flow-based investigation of Cloaking phenomenon among multi-floating bodies and experimental verification
}

\author{
$Z K$ Wang, $G H \mathrm{He}^{*}, Z G$ Zhang, and $Y H$ Meng \\ School of Naval Architecture and Ocean Engineering, Harbin Institute of Technology, Weihai, \\ 264209, China
}

\begin{abstract}
The safety of mooring systems on offshore platforms seems more and more significant with the utilization of offshore space and resources, so the reduction of wave drift force is the key issue in this wavebody interaction problem. The wave drift force acting on the inner floating body surrounded by multiple small floating bodies can be reduced obviously with the occurrence of a phenomenon, which is called the Cloaking phenomenon. The Cloaking phenomenon refers to the reduction or complete elimination in amplitude of the scattered waves. In this paper, a real-coded genetic algorithm was used to optimize the parameters of outer floating bodies to minimize the scattered wave energy, and then the wave drift force acting on the inner body can be reduced. Furthermore, associated CFD simulations and experimental research were conducted with the above optimized parameters to investigate and verify the Cloaking phenomenon more systemically. It is shown that the wave drift force acting on the inner floating body in the Cloaking configuration can be reduced obviously both in numerical and experimental research, and the reduction of the wave drift force is closely related to the change of wave field around the structure.
\end{abstract}

\section{Introduction}

The offshore platform has got a wide range of application with the utilization of ocean energy and the development of large-scale marine structures, and the position system plays a significant role in the stability and safety of the platform. Recently, a Cloaking phenomenon has been investigated and verified in various wave fields, which refers to the reduction or complete elimination in amplitude of the scattered waves. It is known that the wave drift force can be calculated by the amplitude of scattered outgoing waves according to the momentum conservation, so the reduction of wave drift force can be realized with the occurrence of the Cloaking phenomenon in water waves. At this time, the position system of marine structure can be protected by utilizing the Cloaking phenomenon.

The Cloaking phenomenon has been studied in the electromagnetic wave, acoustic wave and seismic wave fields [1-3]. As for the water waves, some research has also been carried out [4-11]. Newman [7,8] successfully realized the Cloaking phenomenon of a truncated

\footnotetext{
* Corresponding author: ghhe@hitwh.edu.cn
} 
cylinder, and found that the total scattered wave energy can become minimal by optimizing the physical dimension of outer floating bodies at a certain frequency, and then the wave drift force acting on the whole array of floating bodies is reduced obviously. Then, Iida et al. [9] demonstrated that the wave drift force can be reduced not only on the whole array of floating cylinders but also on the central floating cylinder. Zhang et al. [10] firstly found the quasi-Cloaking phenomenon of an array with truncated cylinders. Furthermore, Iida et al. [11] investigated the wave pattern in a Cloaking configuration and conducted a model experiment to verify the numerical results.

So far, the investigation on the mechanism about the reduction of wave drift force in a Cloaking configuration from a view of spatial distribution of wave elevation has not been carried out. Following the work of Newman and Iida et al. [7-9], the hydrodynamic forces on the inner floating body in Cloaking configurations and the spatial distribution of wave elevation are investigated in this paper; besides, the relationship between the reduction of wave drift force and the variety of wave surface distribution is also systematically studied.

In this paper, firstly, in order to determine the optimal geometric dimensions of outer floating bodies at the certain frequency which corresponds to the minimal scattered wave energy, a genetic algorithm (GA) is adopted. Then, both CFD numerical simulations and corresponding model experiments are conducted with the above determined geometric parameters at the certain frequency to investigate and verify the Cloaking phenomenon in water-wave field systematically. The spatial distribution of wave elevation around the inner floating cylinder and the wave drift forces are both studied in detail. It is shown that the wave drift force acting on the inner floating body can be reduced to very small not only in numerical results but also in experimental measurements with the occurrence of Cloaking phenomenon and the reduction of the wave drift force is owing to the reduction of wave elevation difference between the weatherside and the leeside of the inner floating body.

\section{Theory}

\subsection{Arrangement of floating structures}

Several outer floating bodies of the same size are placed with equally spaced in a circle of $R$ concentric with the inner floating body and the total number of the outer surrounding bodies equal to $N$. Newman [8] suggested that the scattered wave energy is gradually decreased with the increasing in the number of out floating bodies $N$, and he also demonstrated that the optimized scattered wave energy are practically the same for $N \geq 8$, therefore this paper considers the cases of $N=0$ (inner floating cylinder alone), 4 and 8 . Figure 1 shows the configurations of Cloaking models studied in this paper. The wave incidents from the negative $x$-axis, and the alphabets from a to $\mathrm{j}$ represent the locations of the wave probes. It needs to note that the locations of the wave probes are all the same for $N=0,4$ and 8 . The draft and the diameter of the inner floating cylinder are normalized to unity, that is, $d_{0}=1,2 r_{0}=1.0$. Besides, the draft and the radius of the outer surrounding cylinders are expressed as $d_{\text {out }}$ and $r_{\text {out }}$, which are nondimensionalized with the draft of the inner floating cylinder $d_{0}$.

The present paper uses a genetic algorithm to optimize the geometric parameters of the outer floating bodies $\left(R, r_{\text {out }}, d_{\text {out }}\right)$ to minimize the total scattered wave energy of the whole structures. The optimization process is carried out at the dimensionless wave number $K=$ $1.0\left(K=k d_{0}, k\right.$ represents the wave number).

\subsection{CFD simulations}


The corresponding CFD simulations are conducted to systematically investigate the hydrodynamics performances of the Cloaking configurations with the geometric parameters determined in the section 2.1. The CFD simulations are conducted based on the CFD software FLUENT, which needs to append a UDF (User Defined Function) module to realize the wave-generation and absorption.

The incompressible Navier-Stokes equations and the continuity equation with the velocity and pressure as variables in Cartesian coordinates are adopted as the governing equations to solve the flow problem. The VOF (Volume of fluid) method is adopted to capture the free surface.

The height of wave elevation and particle velocities associated with corresponding fluid fluctuations are specified at an incident boundary to generate waves through further development to the CFD software FLUENT. Besides, the Cartesian coordinates system with its origin at the center of the structure is used in this paper, as shown in figure 1, and the plane of $y=0$ is placed on the undisturbed free surface.

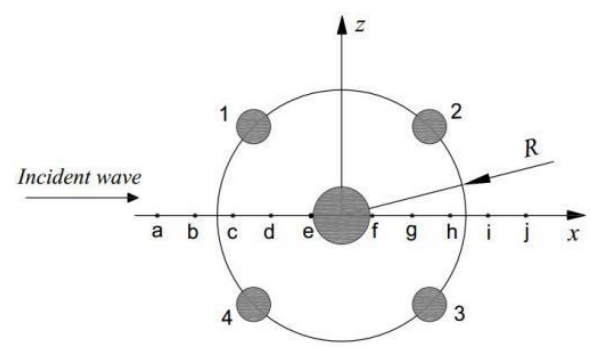

(a) $N=4$

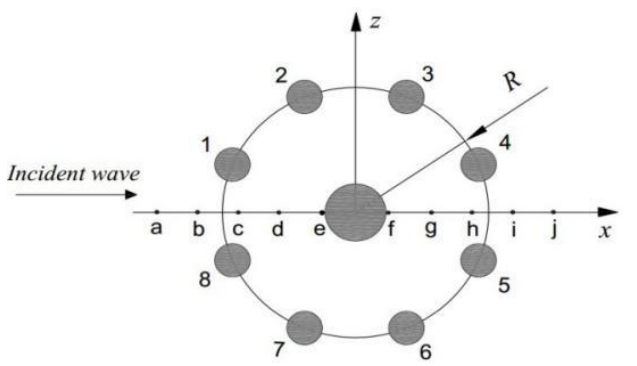

(b) $N=8$

Fig.1. Plan view of the array of multiple floating bodies.

The fluctuation and energy of the wave-absorption region are dissipated by adding source function to the momentum equations to avoid wave reflection from the terminal side of the tank. Limited by the length of the paper, the specific theoretical formulas, systematic mesh convergence test and time step test are not shown here. And the suitable mesh sizes and time step are as follows: mesh size in $x$-axis is $\delta x=L / 50$, which gradually increases in the wave-absorption region to provide additional numerical dissipation and to decrease the CPU time dramatically, mesh size in $y$-axis near the free surface is $\delta y=H / 20$, time step adopted in the present paper is $\delta t=T / 400$.

\subsubsection{Numerical implementation.}

The central difference scheme is used to discrete the diffusion terms, and the convection terms discretization schemes adopted in the present paper are as follows: the Body Force Weighted discretization scheme is used for the pressure, the second-order upwind discretization scheme is used for the momentum, and the Pressure Implicit with Splitting of Operators (PISO) algorithm is used for the pressure-velocity coupling.

\subsection{Model experiments}

The corresponding model experiments are also carried out with the optimization parameters to verify the existence of the Cloaking phenomenon and the accuracy of numerical results. The specific geometric sizes of the array of multi-floating cylinders are presented in the section 3.1. 


\section{Results and discussions}

\subsection{Optimization results}

The draft and the diameter of the inner floating cylinder are normalized to unity, that is, $d_{0}$ $=1,2 r_{0}=1.0$; the draft $\left(d_{\text {out }}\right)$, radius $\left(r_{\text {out }}\right)$ of the outer surrounding cylinders and the distance between the inner and outer surrounding cylinders $(R)$ are optimized using a genetic algorithm so as to minimize the total scattered wave energy of the whole structure, which are all nondimensionalized with the draft of the inner floating cylinder $d_{0}$. The optimization process is carried out at the dimensionless wave number $K=1.0$. And the specific dimensionless optimized geometric parameters are shown in Table 1.

Table 1. Dimensionless geometric parameters obtained by GA.

\begin{tabular}{|l|c|c|c|c|c|}
\hline$N$ & $r_{0}$ & $d_{0}$ & $r_{\text {out }}$ & $d_{\text {out }}$ & $R$ \\
\hline$N=0$ & 0.5 & 1.0 & - & - & - \\
\hline$N=4$ & 0.5 & 1.0 & 0.2909 & 0.3574 & 2.2349 \\
\hline$N=8$ & 0.5 & 1.0 & 0.2909 & 0.4766 & 2.1969 \\
\hline
\end{tabular}

What needs to be explained here is that the draft $\left(d_{\text {out }}\right)$, radius $\left(r_{\text {out }}\right)$ and the separation distance $(R)$ are all optimized in the cases when $N=8$ with the minimization of total scattered wave energy as the objective function, which is called a three-parameter optimization. In order to make use of the outer surrounding cylinders which are already fabricated, the optimization process conducted in the cases when $N=4$ is a two-parameter optimization; that is to say, only the draft $\left(d_{\text {out }}\right)$ and the separation distance $(R)$ are optimized in the cases with $N=4$.

In the CFD simulations and experimental researches, the draft and the radius of the inner floating cylinder selected in the present paper are equal to $d_{0}=0.2234 \mathrm{~m}$ and $r_{0}=$ $0.1117 \mathrm{~m}$, respectively. Besides, the target wave steepness $H / L$ is approximately equal to 0.03 .

\subsection{Wave drift forces and wave elevations}

The wave drift force on the inner floating cylinder can be reduced dramatically with the occurrence of the Cloaking phenomenon, and the variation of wave forces is closely related to the spatial distribution of wave elevations around the structure. So this section investigates the wave drift force acting on the inner floating cylinder with different number of outer floating bodies $N$, and the wave elevations in the weatherside and the leeside of the inner cylinder are systematically investigated. Besides, the relationships between the wave drift force and the wave elevations are also discussed.

Figure 2 shows the wave drift force on the inner floating body with different $N$, and the results obtained through the method of experiments and CFD simulations are both presented.

It can be seen from figure 2 that the wave drift force is reduced with the increase of $N$, which is agree with the conclusions in [7]. And the variation tendencies of wave drift force obtained by these two methods are basically consistent. Anyhow, the wave drift force acting on the inner floating body can be reduced dramatically through surrounding it with multi-floating bodies, and this phenomenon called Cloaking phenomenon can be realized not only in numerical simulations but also the experiments. 


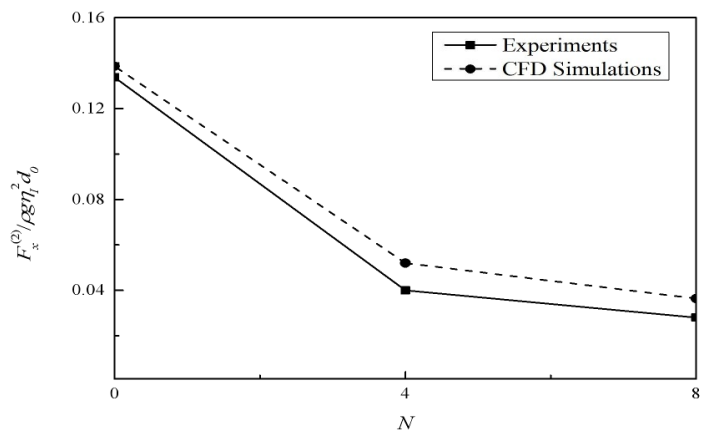

Fig. 2. The wave drift force on the inner floating cylinder at $K=1.0$.

The hydrodynamics forces are affected by the wave elevations around the cylinders, therefore some wave probes are located around the inner floating body in the present paper to measure the wave elevations. The locations of the wave probes are shown in figure 1 . Figure 3 shows the maximum wave elevations in the weatherside and leeside of the inner floating body. The wave elevations at point $a \sim j$ in figure 1 calculated by the CFD method and the elevations at point $\mathrm{e}$ and $\mathrm{f}$ measured through experiments are presented in figure 3 .

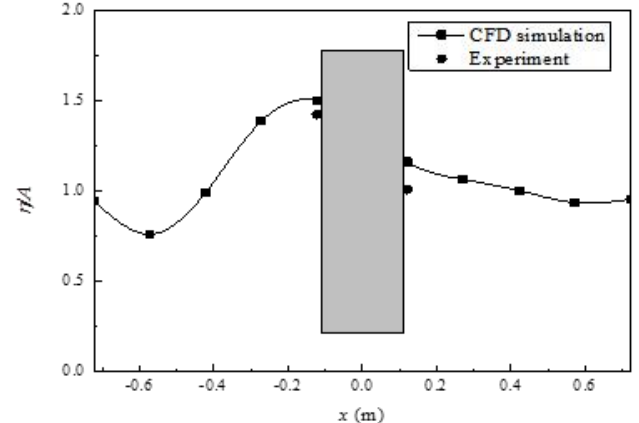

(a) $N=0$

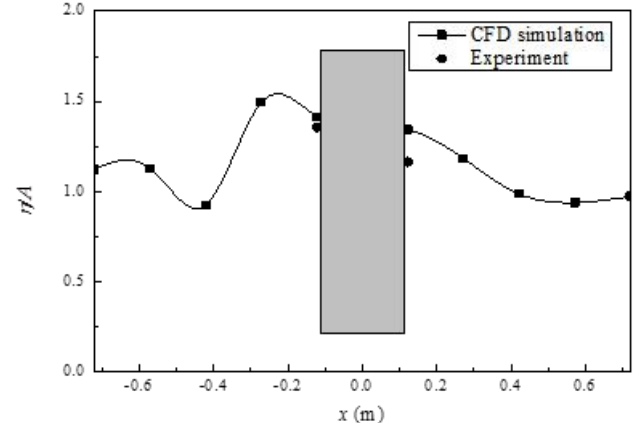

(b) $N=4$

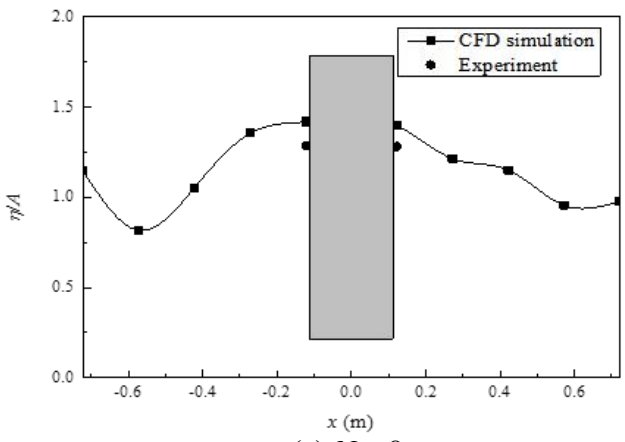

(c) $N=8$

Fig. 3. The wave elevations in the weatherside and leeside of the inner floating body at $K=1.0$.

It can be seen from figure 3 that the wave elevation difference in the weatherside and the leeside of the inner floating body is large in the case of $N=0$, which leads to a large value of wave drift force as shown in figure 2. The wave elevation difference is gradually reduced with the increase of $N$, corresponding to the reduction of wave drift force in figure 2. It should be noted that the wave elevation between the two sides in the case with $N=8$ is even almost the same, which corresponds to the significant reduction of the wave drift force on the inner floating body. And the change low of the wave elevations obtained by the CFD 
simulations and model experiments is practically the same, which indicates the accuracy of the numerical simulations.

\section{Conclusions}

The present paper investigates the Cloaking phenomenon through numerical simulations and experimental measurements. The wave drift force on the inner floating body and spatial distribution of wave elevations around the body are systematically discussed. It is shown that the wave drift force on the inner floating body located in a Cloaking configuration can be reduced dramatically, which can be realized not only in numerical simulations but also in model experiments. Furthermore, the reduction of wave drift force is owing to the reduction of wave elevation difference between the weatherside and the leeside of the inner floating body. Besides, the CFD method is an effective way to study the Cloaking phenomenon of water-waves in detail.

\section{Acknowledgments}

This work was supported by the National Natural Science Foundation of China (11502059) and the Weihai Science and Technology Development Planning (2014DXGJ10).

\section{References}

1. Pendry J B, Schurig D and Smith D R 2006 Controlling electromagnetic fields Science 312 1780-82

2. Zigoneanu L, Popa B I and Cummer S A 2014 Three-dimensional broadband omnidirectional acoustic ground cloak Nature Materials 13(4) 352-55

3. Brûlé S, Javelaud E H, Enoch S and Guenneau S 2014 Experiments on seismic metamaterials: molding surface waves Phys. Rev. Lett. 112(13) 133901

4. Porter R $201126^{\text {th }}$ Int. Workshop on Water Waves and Floating Bodies (Athens) vol 36 pp 1-4

5. Porter R and Newman J N 2014 Cloaking of a vertical cylinder in waves using variable bathymetry J. Fluid Mech. 750 124-43

6. Farhat M, Enoch S, Guenneau S and Movchan A B 2008 Broadband cylindrical acoustic cloak for linear surface waves in a fluid Phys. Rev. Lett. 101(13) 134501

7. Newman J N $201328^{\text {th }}$ Int. Workshop on Water Waves and Floating Bodies (Marseille) pp $157-60$

8. Newman J N 2014 Cloaking a circular cylinder in water waves Eur. J. Mech. B/Fluids 47(5) $145-50$

9. Iida T, Kashiwagi M and $\mathrm{He}$ G H 2014 Numerical confirmation of Cloaking phenomenon on array of floating bodies and reduction of wave drift force Int. J. Offshore Polar Eng. 24(4) 241-46

10. Zhang Z G, He G H, Kashiwagi M and Wang Z K 2018 A quasi-cloaking phenomenon to reduce the wave drift force on an array of adjacent floating bodies Appl. Ocean Res. 71 1-10

11. Iida T, Kashiwagi M and Miki M 2016 Wave pattern in cloaking phenomenon around a body surrounded by multiple vertical circular cylinders Int. J. Offshore Polar Eng. 2015(1) 635-41 